\title{
Radial variations of the SFHs of dwarf irregular galaxies $\dagger$
}

\section{Hong-Xin Zhang ${ }^{1,2,3,4}$, Deidre A. Hunter ${ }^{1}$, Bruce G. Elmegreen ${ }^{5}$, $\mathrm{Yu} \mathrm{Gao}^{2,3}$, and Andreas Schruba ${ }^{6}$}

\author{
${ }^{1}$ Lowell Observatory, 1400 West Mars Hill Road, Flagstaff, Arizona 86001 USA \\ ${ }^{2}$ Purple Mountain Observatory, Chinese Academy of Sciences, 2 West Beijing Road, Nanjing \\ 210008 China \\ ${ }^{3}$ Key Laboratory of Radio Astronomy, Chinese Academy of Sciences, Nanjing 210008 China \\ ${ }^{4}$ Graduate School of the Chinese Academy of Sciences, Beijing 100080 China \\ ${ }^{5}$ IBM T. J. Watson Research Center, PO Box 218, Yorktown Heights, New York 10598 USA \\ ${ }^{6}$ Max-Planck-Institut für Astronomie, Königstuhl 17, 69117 Heidelberg Germany
}

email: hxzhang@lowell.edu; dah@lowell.edu

\begin{abstract}
The LITTLE THINGS project $\dagger$ has compiled multi-wavelength data (including VLA $\mathrm{H}$ I-line emission maps, GALEX FUV/NUV imagery, $U B V$, narrow-band $\mathrm{H} \alpha$, and Spitzer images) for a representative sample of nearby dwarf irregular (dIrr) galaxies. The broadband data are used to constrain the radial variations of the star formation (SF) rate (SFR) averaged over the past $0.1 \mathrm{Gyr}, 1 \mathrm{Gyr}$ and a Hubble time, with a complete library of model SF histories (SFHs). The recent SF of more than $\sim 80 \%$ of the dIrrs in our sample has been concentrated in the inner disk, and the SF in the outer disk has been markedly suppressed. This outside-in shrinking of the star-forming disk leaves a down-bending (double exponential) stellar mass surface density $\left(\Sigma_{\star}\right)$ distribution. Our findings in dIrrs are in contrast to the inside-out disk growth scenario suggested for luminous spiral galaxies.
\end{abstract}

Keywords. galaxies: dwarf - galaxies: irregular - galaxies: evolution - galaxies: stellar content

\section{Introduction}

Dwarf Irregulars (dIrrs) are the most common type of star-forming galaxies in the local universe (e.g. Gallagher \& Hunter 1984). Compared to the larger spiral galaxies, gas-rich dIrrs are smaller, bluer, metal-poor, and less luminous. In the classical "insideout" growth mode, the disk scale length increases with time due to the slower/later accretion of gas at large radii and exhaustion of gas in the centers (e.g. Larson 1976). The negative color gradients and abundance gradients observed in typical spiral galaxies fit well into the inside-out disk growth scenario. However, late-type dIrrs usually show more or less flat gradients of current metallicity (e.g. Croxall et al. 2009), implying the inside-out paradigm may not apply to dIrrs.

Lack of large-scale instabilities (Hunter et al. 1998), such as spiral arms and bars, in dIrrs means that the present-day stellar population distribution is primarily determined by the in situ SFHs, rather than a redistribution process (e.g. resonant scattering with

$\dagger$ Based on data from the LITTLE THINGS Survey (Hunter et al., in preparation), funded in part by the National Science Foundation through grants AST-0707563, AST-0707426, AST-0707468, and AST-0707835 to US-based LITTLE THINGS team members and with generous support from the National Radio Astronomy Observatory.

$\dagger$ Local Irregulars That Trace Luminosity Extremes - The H I Nearby Galaxy Survey, http://www.lowell.edu/users/dah/littlethings/index.html 
spiral arms) following in situ SF. This makes it possible to probe the stellar disk formation process by studying the present-day stellar population distribution across the disk.

To understand the disk growth process of dIrrs, we derived radial variations of the azimuthal averages of the multi-band spectra energy distributions (SEDs) (i.e. GALEX FUV/NUV, $U B V$, narrow-band $\mathrm{H} \alpha$, and Spitzer $3.6 \mu \mathrm{m}$ ) for 34 dIrrs selected from the LITTLE THINGS sample (41 galaxies). The galaxies studied in this work are selected to have the full complement of data available. These surface brightness profiles are fit with stellar population synthesis models. Considering the complex SFHs found in local dIrrs (e.g. Weisz et al. 2011), we construct a relatively complete SFH library by dividing the Hubble time into 6 independent age bins, where each bin is assumed to have a constant SFR (see Zhang et al. 2011, hereafter Z11).

\section{Results}

Figure 1 presents the main results of 4 typical galaxies included in our sample. The readers are referred to $\mathrm{Z} 11$ for the results of the whole sample. From the left column of Figure 1, we can see that all four galaxies exhibit obvious breaks in their surface brightness profiles. The blue compact dwarf (BCD) galaxy Haro 29 shows significant excess starlight in the central regions in all of the bands. This suggests that the central regions have sustained the starburst for an extended period of time. The multi-band surface brightness profiles exhibit different radial variations among different bands interior to the break radius, beyond which the shorter wavelength profiles fall off faster than the longer wavelength profiles. Actually, more than $\sim 80 \%$ of the whole sample show a steeper radial decline of the surface brightness profiles in the outer disk.

As is shown in the middle column of Figure 1, recent SF is concentrated in the inner disk. For all the galaxies shown here except Haro 29, the radial variations of recent $\mathrm{SF}$ follow the lifetime average $\mathrm{SF} \Sigma_{\mathrm{SFR} \mathrm{R}_{13.7 \mathrm{Gr}}}$ much better in the inner disk than in the outer disk. The ratios $\Sigma_{\mathrm{SFR}_{0.1 \mathrm{G} \mathrm{yr}}} / \Sigma_{\mathrm{SFR}_{13.7 \mathrm{Gyr}}}$ and $\Sigma_{\mathrm{SFR}_{1 \mathrm{G} \mathrm{yr}}} / \Sigma_{\mathrm{SFR}_{13.7 \mathrm{Gr}}}$ exhibit steep declines beyond the break radius. The steeper radial decline of $\Sigma_{\mathrm{SFR}_{0.1 \mathrm{Gyr}}} / \Sigma_{\mathrm{SFR}_{13.7 \mathrm{~Gy}}}$ and $\Sigma_{\mathrm{SFR}_{1 \mathrm{Gyr}}} / \Sigma_{\mathrm{SFR}_{13.7 \mathrm{Gr}}}$ in the outer disk was found for more than $\sim 80 \%$ of our whole sample (Figures 6 and 7 in Z11). Therefore, in contrast with the randomly percolating SF scenario suggested for dIrrs (e.g. van Zee 2001), the star-forming disks of dIrrs has been shrinking.

The right column of Figure 1 shows the $\Sigma_{\star}$ profiles derived from our SED modeling. The $\Sigma_{\star}$ profiles are well fitted with either a single or piece-wise exponential function, which is overplotted on the profiles. Except for Haro 29, which has an up-bending $\Sigma_{\star}$ profile beyond the break, the other three galaxies have down-bending $\Sigma_{\star}$ profiles. About $80 \%$ of our whole sample of galaxies exhibit obvious down-bending $\Sigma_{\star}$ profiles (Z11). One important difference between our dIrrs and spiral galaxies is that, a down-bending surface brightness profile usually accompanies a down-bending $\Sigma_{\star}$ profile, whereas in spiral galaxies the break of a down-bending surface brightness profile is almost gone in the $\Sigma_{\star}$ profile. For dIrrs, the breaks in the radial profiles of $\Sigma_{\mathrm{SFR}_{0.1 \mathrm{Gyr}}} / \Sigma_{\mathrm{SFR}_{13.7 \mathrm{G} y \mathrm{r}}}$ and $\Sigma_{\mathrm{SFR}_{1 \mathrm{Gyr}}} / \Sigma_{\mathrm{SFR}_{13.7 \mathrm{G} y \mathrm{r}}}$ come with breaks in the $\Sigma_{\star}$ profiles.

\section{Discussion}

On average, SF in outer disks has been decreasing more significantly both over time and over radius (Figure 1) compared to inner disks, which would naturally lead to downbending stellar mass surface density profile, seen in most of our galaxies. This scenario of outside-in depression of SF is different from what may be happening in typical BCD 

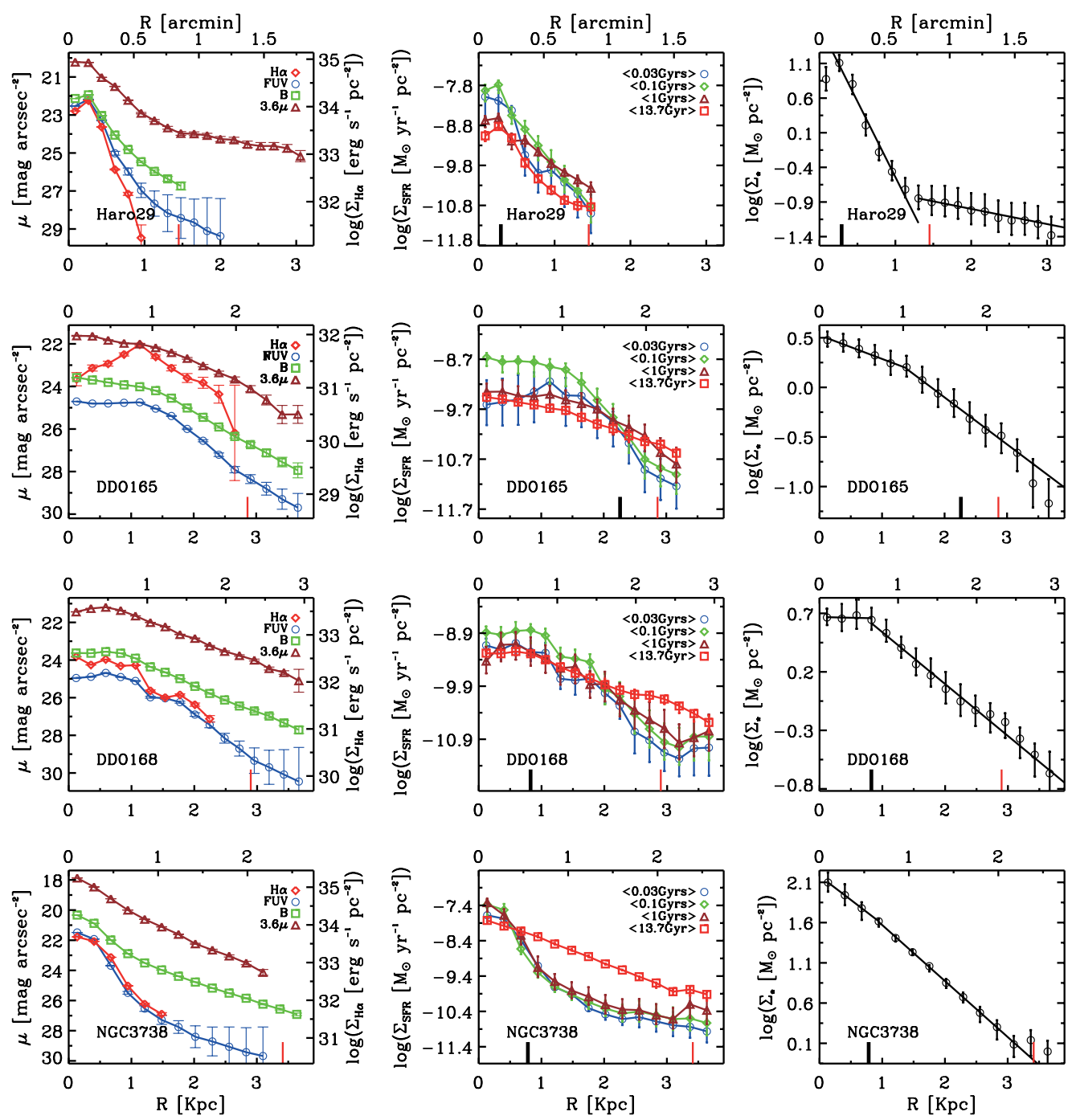

Figure 1. First column: Azimuthally-averaged surface brightness profiles of $\mathrm{H} \alpha, \mathrm{FUV}, B$ and $3.6 \mu \mathrm{m}$ images. The NUV or $U$-band profiles are plotted instead of FUV if there are no FUV observations. Second column: Radial variations of the SFR averaged over different timescales obtained from our multi-band SED modeling. The averaging timescales are the most recent 0.03 Gyr, the recent $0.1 \mathrm{Gyr}, 1 \mathrm{Gyr}$, and the Hubble time. Third column: Inclination-corrected $\Sigma_{\star}$ profiles from the SED modeling. The fitted exponential profiles are overplotted as solid lines. The thin vertical line marks the Holmberg radius, and the thick black vertical line marks the $V$-band scale length.

(e.g. Haro 29) galaxies. The central starburst of BCD galaxies results in faster buildup of the inner stellar disk than the outer part, which could lead to up-bending $\Sigma_{\star}$ profiles.

In agreement with previous findings in a few nearby dIrrs (e.g. McConnachie et al. 2006; Bernard et al. 2007; Gallart et al. 2008), the star-forming disk of our sample of dIrrs is experiencing outside-in shrinking. The low mass of dIrrs suggests that they are susceptible to both environmental influences (e.g. ram pressure stripping of gas caused by the intragroup or intracluster medium and interactions with neighboring galaxies) and regulation through stellar feedback. The outer disk, especially in relatively low mass 
systems where the gravitational well and the gas density are relatively low, is more prone to gas removal due to ram pressure stripping. Z11 (their Figure 10) showed that galaxies with a larger tidal index, and hence more likely to have been influenced from the outside, (Karachentsev et al. 2004) prefer lower (zero or negative) slopes in radial variations of $\log \left(\Sigma_{\mathrm{SFR}_{0.1 \mathrm{G} \mathrm{yr}}} / \Sigma_{\mathrm{M}_{\star}}\right)$ and $\log \left(\Sigma_{\mathrm{SFR}_{1 \mathrm{G} \mathrm{yr}}} / \Sigma_{\mathrm{M}_{\star}}\right)$. Numerical simulations (e.g. Kapferer et al. 2008) suggest that both ram pressure stripping and tidal disturbance are needed to reproduce the observations of dIrrs.

\section{References}

Bernard E. J., Aparicio A., Gallart C., Padilla-Torres C. P., \& Panniello M. 2007, AJ, 134, 1124 Croxall, K. V., van Zee, L., Lee, H., et al. 2009, ApJ, 705, 723

Gallagher, J. S. \& Hunter, D. A. 1984, ARAA, 22, 37

Gallart, C., Stetson, P. B., Meschin, I. P., Pont, F., \& Hardy, E. 2008, ApJ, 682, 89

Hunter, D. A., Elmegreen, B. G., \& Baker, A. L. 1998, ApJ, 493, 595

Kapferer, W., Kronberger, T., Ferrari, C., Riser, T., \& Schindler, S. 2008, MNRAS, 389, 1405

Karachentsev, I. D., Karachentseva, V. E., Huchtmeier,W. K., \& Makarov, D. I. 2004, AJ, 127, 2031

Larson, R. B. 1976, MNRAS, 176, 31

McConnachie, A. W., Arimoto, N., Irwin, M., \& Tolstoy, E. 2006, MNRAS, 373, 715

van Zee, L. 2001, AJ, 121, 2003

Weisz, D. R., Dalcanton, J. J., Williams, B. F., et al. 2011, ApJ, 739, 5

Zhang, H.-X., Hunter, D. A., Elmegreen, B. G., Gao, Y., \& Schruba, A. 2011, arXiv: 1111.3363 (Z11)

\section{Discussion}

AquAVIVA: What is the star formation history reconstructed in the six bins in the Hubble time?

ZHANG: According to the test of the reliability of our SED modelling, only the starformation rate (SFR) averaged over the past 0.1 Gyr, 1 Gyr and the Hubble time could be well constrained. This is because we only have a few broadband data points. Therefore, we only focus on the interpretation of the SFR averaged over the 0.1 Gyr, 1 Gyr and the Hubble time. 